\title{
Mesophase Structure discovered through in-situ X-ray measurement in drawing process of poly(ethylene 2,6-naphthalene dicarboxylate) fiber
}

\author{
KyoungHou Kim ${ }^{1}$, Ryo Aida ${ }^{2}$, YoungAh Kang, ${ }^{2}$ Yutaka Ohkoshi ${ }^{2 *}$, Yasuo Gotoh², Masanobu Nagura ${ }^{2}$, Hiroshi \\ Urakawa $^{3}$ \\ ${ }^{1}$ Collaborative Innovation Center for Nanotech FIBER (nanoFIC), Shinshu University, Ueda, Nagano 386-8567, \\ Japan \\ ${ }^{2}$ Faculty of Textile Science and Technology, Shinshu University, 3-15-1 Tokida, Ueda, Nagano 386-8567, Japan \\ ${ }^{3}$ Graduate School of Science and Technology, Kyoto Institute of Technology, Goshokaidoucho, Matsugasaki, \\ Sakyo-ku, Kyoto 606-8585, Japan
}

The structure development in the continuous laser-heated drawing process of poly(ethylene 2,6-naphthalene dicarboxylate) (PEN) fiber was analyzed by in-situ X-ray diffraction measurement. Because of the rapid and uniform laser-heating, and the resultant steady-state nature of the necking-drawing, the structure development after the on-set of necking could be measured in the time resolution of several hundred microseconds. We found for the first time the temporal appearance of meridional (001') diffraction at several milliseconds after the on-set of necking indicating that the mesophase structure similar to the one reported for poly(ethylene terephthalate) was also formed in the initial stage of fiber structure development of PEN. The d-spacing of the (001') diffraction $1.230 \pm$ $0.003 \mathrm{~nm}$ was shorter than the c-axis lengths of both $\alpha$ and $\beta$ crystals.

\section{Introduction}

Poly(ethylene 2,6-naphthalene dicarboxylate) (PEN), containing naphthalene ring in its backbone, possesses higher glass-transition temperature $\left(\mathrm{T}_{\mathrm{g}}\right)$ and higher melting temperature $\left(\mathrm{T}_{\mathrm{m}}\right)$ than those of poly(ethylene terephthalate) (PET). Due to the stiff molecular chain of PEN, it can be expected to give high-performance fibers having excellent physical properties for engineering uses.

There have been studied on hot-drawing and cold-drawing processes in PEN fibers and films, ${ }^{[1,2]}$ in which the drawability of amorphous PEN was reported to depend on molecular weight and strain rate. The structural changes in the neck-drawing of amorphous PEN films have been investigated through off-line measurement using synchrotron X-ray radiation, ${ }^{[1,3]}$ which revealed the appearance of smectic order with a period of $1.25 \mathrm{~nm}$ corresponding to a chain repeat length associated with a sinusoidal conformation of the polymer chains. It was $5 \%$ shorter than the chain repeat length of a more extended $\alpha$-conformation. However, so far, there has been found no report about on-line characterization of structure development process with orientation-induced crystallization in PEN fiber and films.

Several studies on the on-line characterization of the fiber structure development during the drawing have been conducted using simultaneous synchrotron radiation as well as laboratory X-ray generators for WAXD and SAXS. ${ }^{[4-11]}$ Many of these studies concerned PET. Our research group has studied on the fiber structure development process in the continuous laser-drawing for PET and PTT fibers through on-line WAXD and SAXS measurements ${ }^{[9]}$ with synchrotron radiation systems ${ }^{[10,11,12]}$. Quantitative analyses on the fiber structure development mechanism were accomplished by direct measurement in the vicinity of the neck-deformation point, in which drawing behavior, fiber temperature changes, and WAXD/SAXS were measured as a function of elapsed time after the neck-deformation. ${ }^{[9-12]}$ The elapsed time, calculated from distance between measurement point and the neck-deformation point, is high accuracy because the neck point could be fixed within confined region by rapid and uniform heating of a running fiber due to the $\mathrm{CO}_{2}$ laser radiation. We have analyzed quantitatively the fiber structure development by the on-line measurement with time resolutions $1 \mathrm{~ms}$ for X-ray diffraction measurement and $0.47 \mathrm{~ms}$ for fiber temperature profile on the PET running fiber.

In this study, the fiber structure development of PEN fiber in the neck-drawing was investigated using our laser-drawing system and on-line measurement techniques. 


\section{Experimental}

\section{Fundamentals of On-line Measurement}

Fig. 1 showed a schematic diagram of the on-line measurement system used in this study. Details of the on-line system can be found in our previous papers. ${ }^{[10-12]}$ The running fiber was heated by $\mathrm{CO}_{2}$ laser beam generated with a PIN-20S laser source manufactured by Onizca Glass Co. Ltd. The laser source was $20 \pm 1 \mathrm{~W}$ in rated power and $5 \mathrm{~mm}$ in beam diameter. The fiber was drawn by means of a speed difference between feed and take-up rollers. Control of the neck-deformation point within 1 $\mathrm{mm}$ by laser irradiation maintained well-stable and well-steady state for the neck-drawing. X-ray diffraction images were recorded as a function of elapsed time $t$, which was calculated from the distance $x$ between the measurement point and the neck-deformation point, divided by fiber running speed $v$. The distance $x$ was changed by shifting the neck-deformation point with traveling mirror unit.

Fig. 2 showed a neck-deformation behavior taken by CCD camera during the neck-drawing of PEN fiber. It appeared a long neck-drawing region, which is presumably due to low drawing stress of 28 $\mathrm{MPa}$. The CCD camera image of $4.7 \mathrm{~mm}$ in width, corresponding to 640 pixels, has a resolving power of $0.0073 \mathrm{~mm} /$ pixel. The time resolution of the on-line measurement was $1.5 \mathrm{~ms}$, which was calculated from the diameter of X-ray beam $0.20 \mathrm{~mm}$, the fluctuation of neck-deformation point 0.45 $\mathrm{mm}$, and the length of neck-deformation region $0.54 \mathrm{~mm}$, divided by the fiber running speed 0.50 $\mathrm{m} / \mathrm{s}$.

\section{Materials and Drawing Condition}

PEN pellets having intrinsic viscosity $0.94 \mathrm{dl} / \mathrm{g}$ were spun with take-up speed of $390 \mathrm{~m} / \mathrm{min}$, maintaining mass flow rate of $4.0 \mathrm{~g} / \mathrm{min}$. As-spun PEN monofilament of $100 \mu \mathrm{m}$ in diameter was fed $5.46 \mathrm{~m} / \mathrm{min}$ and drawn to the draw ratio of 5.5, where the drawing stress $28 \mathrm{MPa}$ was applied.

\section{X-ray Diffraction}

X-ray diffraction measurements were carried out with synchrotron radiation of BL40B2 in SPring-8, where X-ray beam of $0.1 \mathrm{~nm}$ in wavelength and $200 \mu \mathrm{m}$ in diameter was applied. The X-ray diffraction images were taken for exposure time $8 \mathrm{~min}$ by imaging plate of $3000 \times 3000$ pixel attached to the vacuum chamber. The camera length calibrated by lead stearate $(\mathrm{StPb})$ was $292.72 \mathrm{~mm}$. The intensity profile containing diffraction by polyimide film mounted on the vacuum chamber was used as a reference profile by subtracting blank image.

\section{Results and discussion}

Fig. 3 showed wide-angle X-ray diffraction images taken on the drawing of PEN fiber. Before the neck point, at $-2.5 \mathrm{~ms}$, non-oriented amorphous halo was observed. At $0 \mathrm{~ms}$ and after, the amorphous hallo of smeared image was concentrated to the equatorial direction. X-ray images after the neck point appeared a sharp meridional diffraction, which was not observed for the drawn PEN fiber. The meridional diffraction shows the evidence of mesophase structure formation. Because the mesophase diffraction is typically observed at $2.0 \mathrm{~ms}$ which is longer than the time resolution the mesophase structure was certainly formed after neck-deformation. At $5 \mathrm{~ms}$ after necking, crystal diffractions began to be observed. Although the drawing stress is rather low, the drawn fiber revealed high crystal orientation and high crystallinity.

The meridional diffraction observed from 0 to $5 \mathrm{~ms}$ after necking is just like the (001') diffraction reported for PET ${ }^{[6,7]}$. Our research group also has reported the (001') diffraction by on-line analysis ${ }^{[9,10]}$. Considering a morphological similarity of PEN and PET, the meridional diffraction can be assigned as the (001') diffraction of smectic-like structure as reported for PET. So far, several studies have reported that the mesophase structure was formed after cold-drawing of PEN ${ }^{[1,3]}$. Jakeways et al. ${ }^{[1]}$ have reported the appearance of (001') diffraction for as-spun PEN fiber, but there has not been the mesophase structure by on-line measurement. The clear (001') diffraction firstly found in this study is the important evidence of the mesophase formation in the fiber structure development process of PEN. In contrast, the (001') diffraction was not observed for PTT ${ }^{[11]}$. It was presumably due to the difference of crystallization induction time. The crystal diffraction for PEN was observed from $5 \mathrm{~ms}$ after neck-deformation. In the previous studies, the crystal diffraction started 
to appear at $0.6 \mathrm{~ms}$ for PET, and it appeared just after necking for PTT. It is clear that PEN needs longer transition time from mesophase to crystal than both PET and PTT.

Fig. 4 showed the WAXD intensity profiles in equatorial (a) and meridional (b) directions. The meridional (001') diffraction started to appear at $0 \mathrm{~ms}$, increased the intensity, and almost disappeared at $5 \mathrm{~ms}$. At the same time, the crystal diffractions emerged. Thus, the mesophase structure began to be developed just after the necking, and the crystalline structure should be formed from the mesophase.

The (001') d-spacing calculated by Bragg's equation subsequent to peak-fitting using Gaussian function, was $1.23 \pm 0.003 \mathrm{~nm}$, which was obtained from the first diffraction revealed in the meridian profile. It was somewhat smaller than the reported data $1.25 \mathrm{~nm}^{[3]}$ estimated from the second to the sixth diffractions of cold-drawn film, which has indicated the appearance of smectic order with a period of $1.25 \mathrm{~nm}$ corresponding to a chain repeat length associated with a sinusoidal conformation of the polymer chains, $5 \%$ shorter than that repeat length consisting in molecular chains showing the more extended $\alpha$-conformation. The d-spacing $1.23 \pm 0.003 \mathrm{~nm}$ was much shorter than the c-axis lengths of both $\alpha$-crystal $(1.32 \mathrm{~nm})$ and $\beta$-crystal $(1.273 \mathrm{~nm})$. It indicated that the molecular chain in

the mesophase structure is more shrunken than the molecular conformation in crystal ${ }^{[14,15]}$. In contrast, the (001') d-spacing of PET was 1.064-1.068 nm, which was about $1 \%$ shorter than the c-axis length $(1.075 \mathrm{~nm})$. Thus, the extending of d-spacing for PEN during crystallization was higher than for PET. It may be caused by more molecular chain extension and/or smaller molecular tilting during crystallization.

The equatorial diffractions observed at $2 \theta=9.7^{\circ}$ should be assigned as the $(020)$ of $\beta$-crystal, which confirmed the $\beta$-rich structure of the drawn fiber. It was known that the $\beta$-crystal possesses higher density and shorter c-axis than the $\alpha$-crystal. And it has been reported that the $\beta$-crystal was formed by high-speed spinning or by high-temperature annealing. ${ }^{[16,17]}$ It is interesting that the $\beta$-rich crystal was developed by laser drawing.

\section{Acknowledgements:}

The synchrotron radiation experiments were performed at the BL40B2 in the SPring- 8 with the approval of Japan Synchrotron Radiation Research Institute (JASRI) (2007B1226). This research was supported by a Grants-in-Aid No. 18550191 from the Japan Society for the Promotion of Science. Also, this work was supported by "Innovation Creative Center for Advanced Interdisciplinary Research Areas (Shinshu university)" project in special coordination funds for promoting science and technology of the ministry of education, culture, sports, science and technology, the Japanese government.

\section{Reference}

[1] R. Jakeways, J. L. Klein, and I. M. Ward, Polymer 1996, 37, 3761-3762.

[2] G. E. Welsh, D. J. Blundell, A. H. Windle, J. Mat. Sci. 2000, 35, 5225.

[3] M. C. Garc1'a Gutie'rrez, J. Karger-Kocsis, and C. Riekel, Macromolecules 2002, 35, 7320.

[4] J. Spruiell, J. White, Polym. Eng. Sci. 1975, 15, 660.

[5] H. Haberkorn, K. Hahn, K. Breuer, J. Appl. Polym. Sci. 1993, 47, 1551.

[6] B. S. Hsiao, A. D. Kennedy, R. A. Leach, B. Cho, P. Harney, J. Appl. Cryst. 1997, 30, 1084.

[7] S. Ran, Z. Wang, C. Burger, B. Chu, B. S. Hsiao, Macromolecules 2002, 35, 10102.

[8] J. Wu, J. M. Schultz, J. M. Samon, A. B. Pangelinan, H. H. Chuah, Polymer 2001, 42, 7161.

[9] T. Yamaguchi, K Komoriyama, Y. Ohkoshi, H. Urakawa, Y. Gotoh, N. Terasawa, M. Nagura, K. Kajiwara, J. Polym. Sci.: Part B: Polym. Phys. 2005, 43, 1090.

[10] T. Yamaguchi, K.H. Kim, T. Murata, M. Koide, S. Hitoosa, H. Urakawa, Y. Ohkoshi, Y. Gotoh, M. Nagura, M. Kodera, K. Kajiwara, J. Polym. Sci.: Part B: Polym. Phys. 2008, 46, 2126.

[11] K. H. Kim, Y. A. Kang, T. Murata, S. Ikehata, Y. Ohkoshi, Y. Gotoh, N. Nagura, M. Koide, H. Urakawa, M. Kotera, Polymer 2008, 49, 5705.

[12] K. H. Kim, T. Yamaguchi, Y. Ohkoshi, Y. Gotoh, M. Nagura, H. Urakawa, M. Kotera, T. Kikutani, J. Polym. Sci.: Part B: Polym. Phys. 2009, 47, in press.

[13] W. Okumura, T. Kanegae, Y. Ohkoshi, Y. Gotoh, M. Nagura, Int. Polym. Proc. 2003, 18, 1.

[14] Z. Mencik, Chem. Prumysl. 1967, 17, 78. 
[15] H. G. Zachmann, D. Wiswe, R. Gehrke, C. Riekel, Makromol. Chem. Suppl. 1985, 12.

[16] J. Jager, J. A. Juijin, C. J. M. Van den Heuvel, R. A. Huijts, J. Appl. Polym. Sci. 1995, 57, 1429.

[17] K. Miyata, T. Kikutani, N. Okui, J. Appl. Polym. Sci. 1997, 65, 1415. 
Elapsed time $t=\frac{\text { Distance } x}{\text { Fiber velocity } v}$

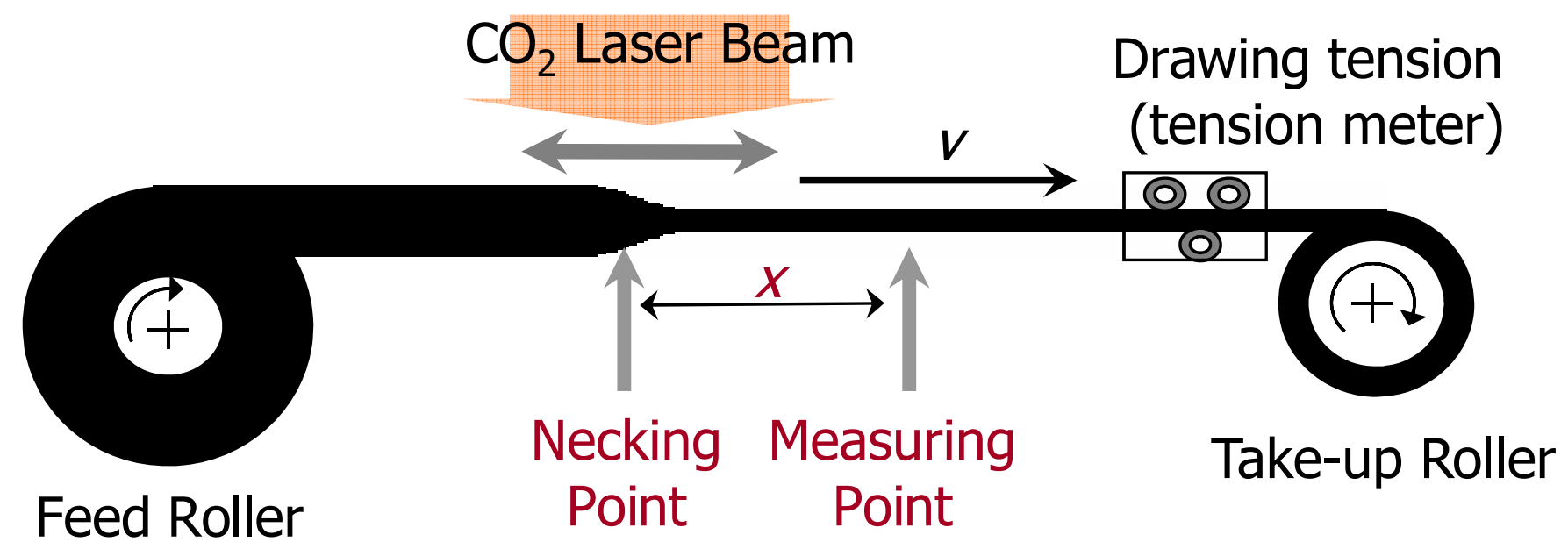

Fig. 1 Schematic diagram of on-line measurement system. 


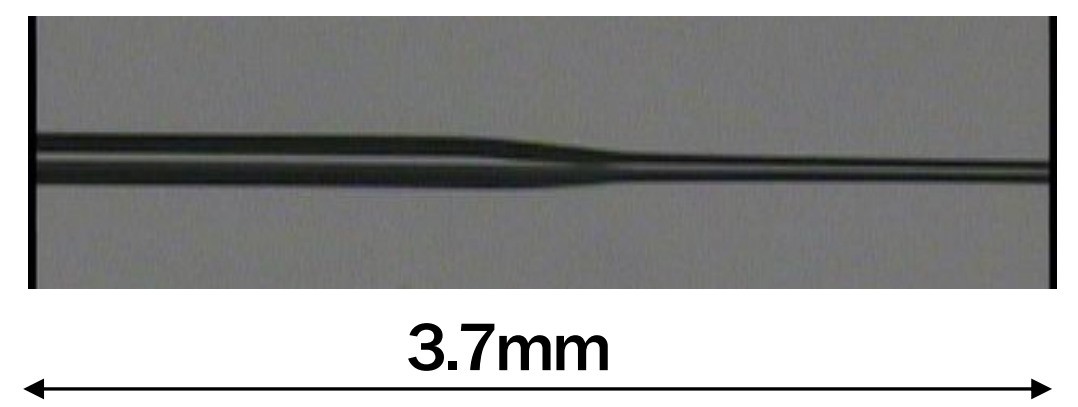

Fig. 2 CCD image in the vicinity of neck-drawing point. 

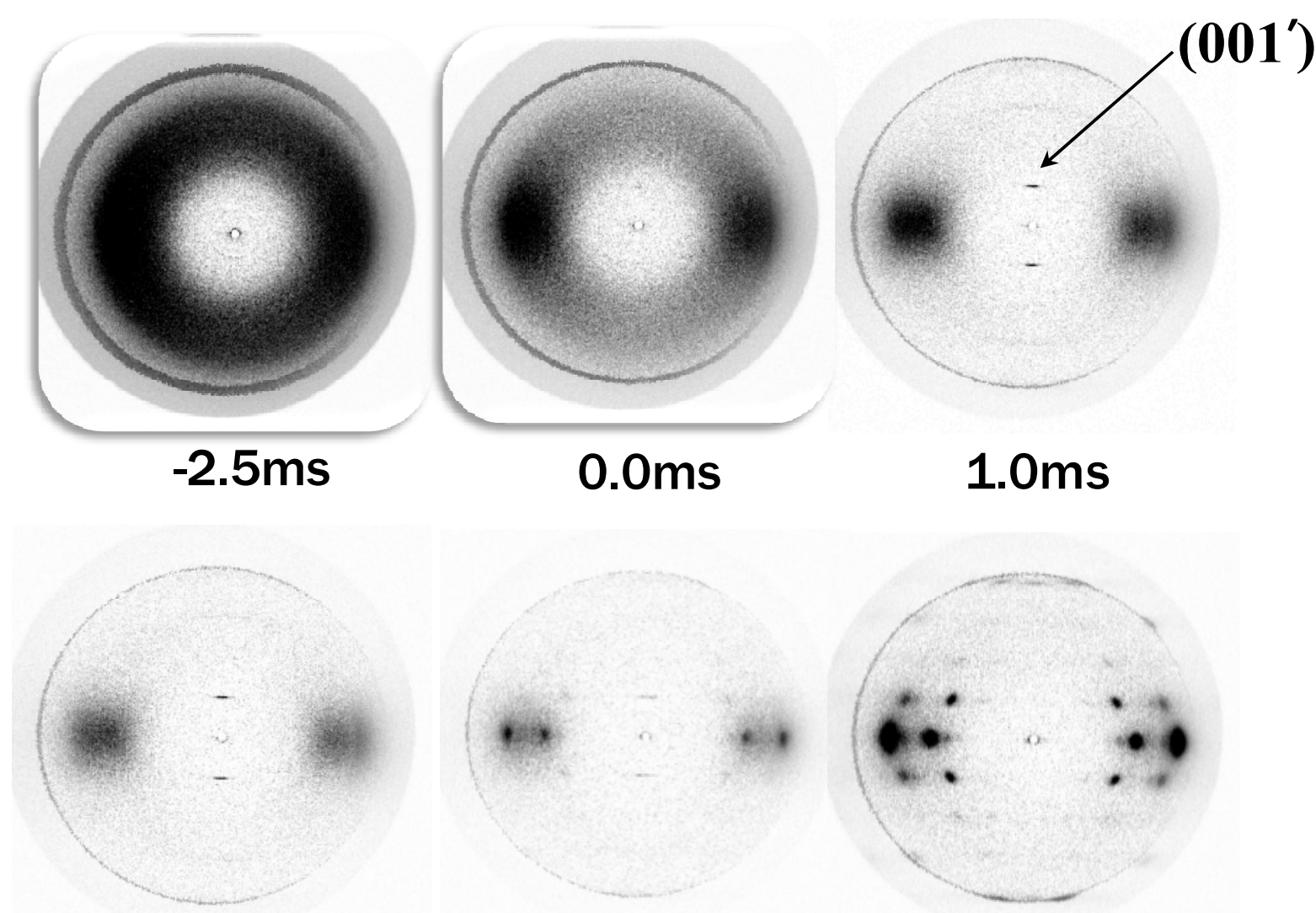

$2.0 \mathrm{~ms}$

$5.0 \mathrm{~ms}$

Drawn Fiber

Fig. 3 WAXD patterns of PEN fiber on the laser drawing-line. The elapsed times are noted below each pattern. 


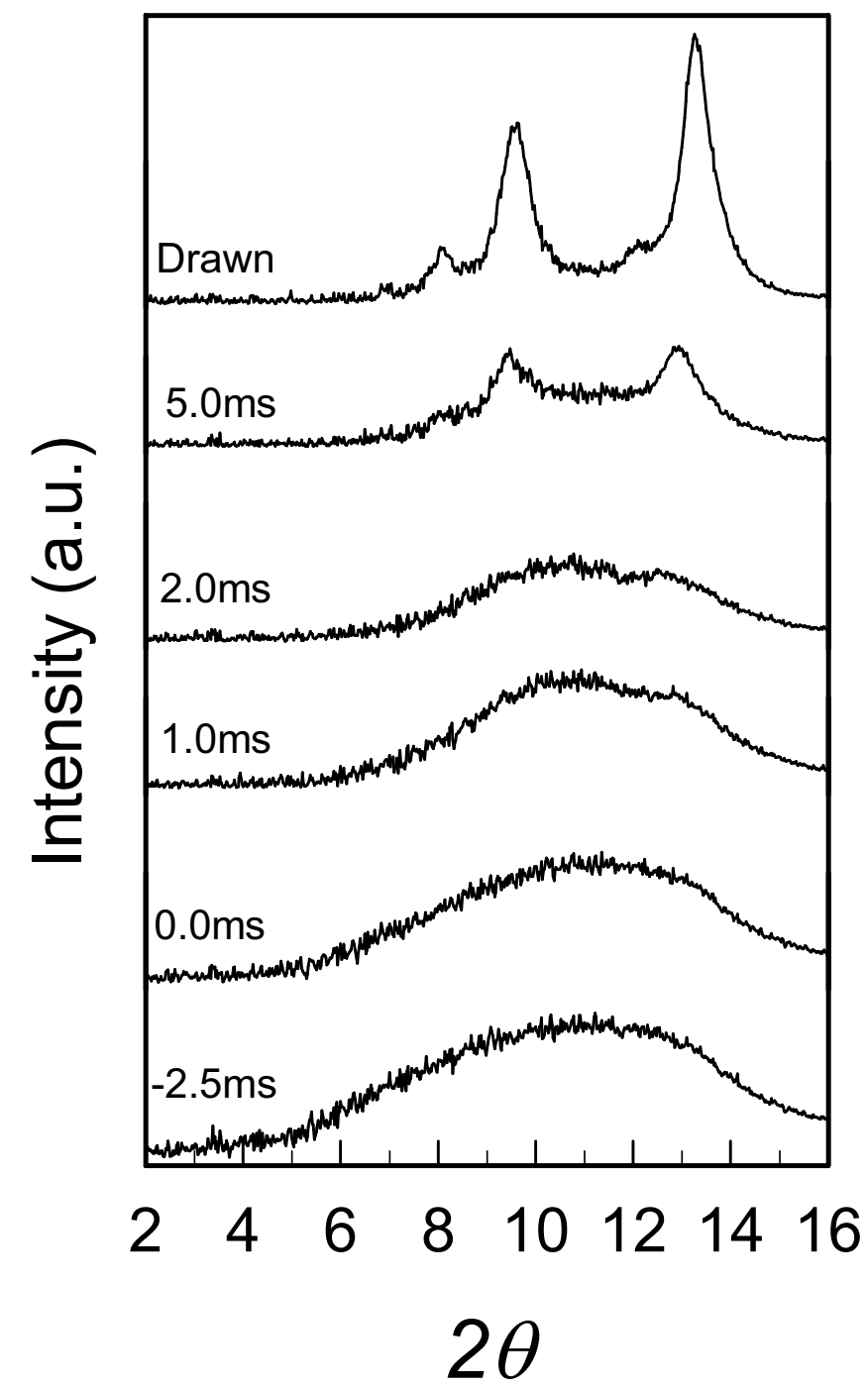

(a)

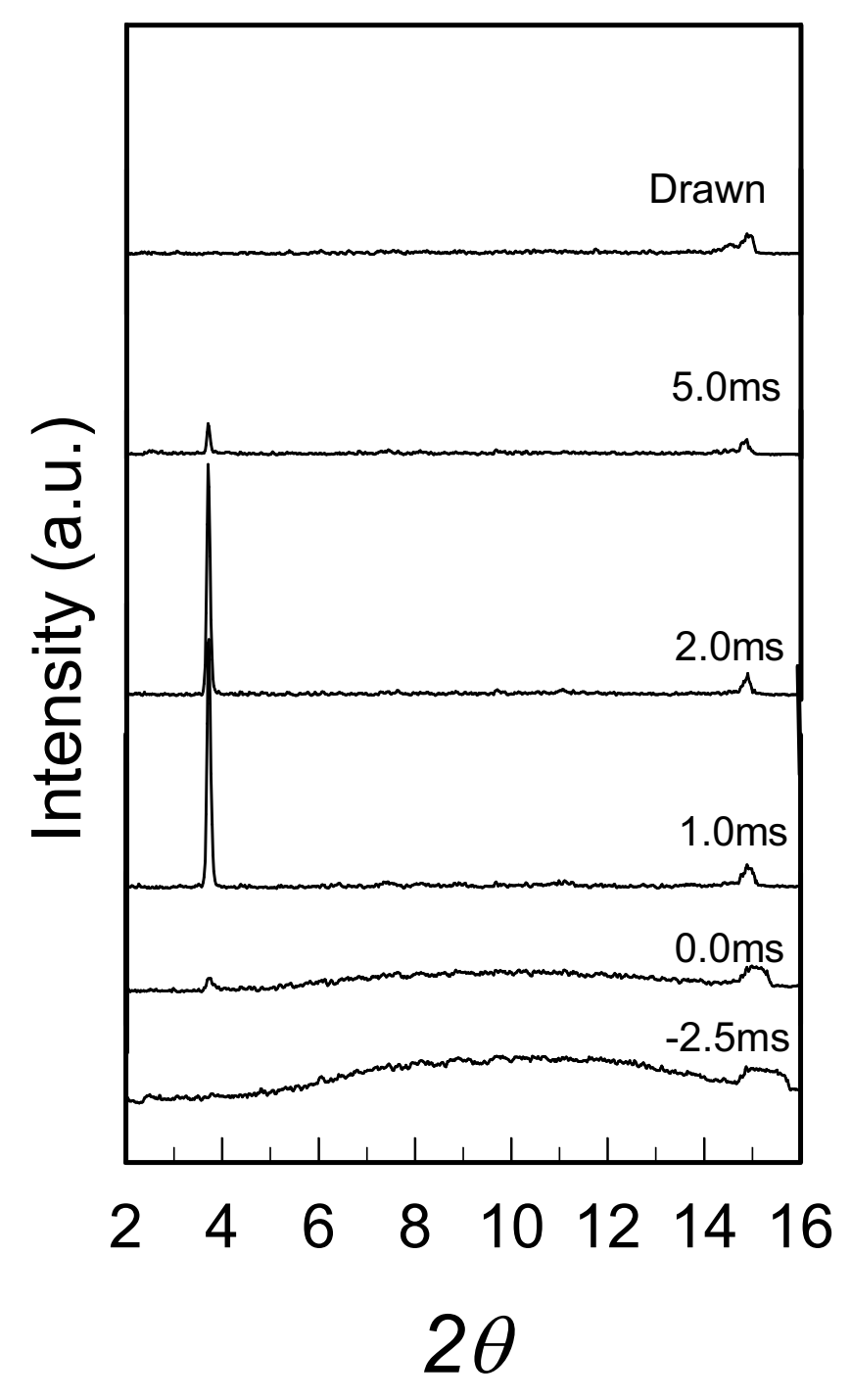

(b)

Fig. 4 X-ray intensity profiles of PEN fiber: (a) equatorial and (b) meridional directions. The elapsed times were noted in the figure. 